\title{
LA CONFISCACIÓN DE ÓRGANOS A LA LUZ DEL DERECHO CONSTITUCIONAL A LA PROTECCIÓN DE LA SALUD'
}

Organ conscription in the light of the constitutional right to health protection

\author{
CLARA MOYA GUILLEM \\ Universidad de Alicante \\ clara.mg@ua.es \\ DAVID RODRÍGUEZ-ARIAS \\ Universidad de Granada \\ dra@ugr.es \\ MARINA MORLA GONZÁLEZ \\ (Universidad de León) \\ mmorlg@unileon.es \\ ÍNIGO DE MIGUEL BERIAIN \\ (Universidad del País Vasco) \\ inigodemiguelb@ehu.eus \\ ALBERTO MOLINA PÉREZ \\ (IESA-CSIC) \\ amolina@iesa.csic.es \\ IVÁN ORTEGA-DEBALLON \\ (Universidad de Alcalá) \\ ivan.ortega@uah.es
}

Cómo citar/Citation

Moya Guillem, C., Rodríguez-Arias, D., Morla González, M. De Miguel Beriain, Í., Molina Pérez, A., Ortega-Deballon, I. (2021).

La confiscación de órganos a la luz del derecho constitucional a la protección de la salud. Revista Española de Derecho Constitucional, 122, 183-213. doi: https://doi.org/10.18042/cepc/redc.122.06

\footnotetext{
Esta investigación se ha realizado como parte del proyecto INEDyTO (Investigación en Ética de la Donación y el Trasplante de órganos) financiado por el Gobierno español (MINECO FFI2017-88913-P) y de la Grant IT-1066-16, financiada por el Gobierno del País Vasco.
} 


\section{Resumen}

En este trabajo analizamos los argumentos a favor y en contra del que hemos denominado modelo de obtención automática en relación con los órganos de fallecidos. Para ello aportamos datos empíricos que permiten valorar su potencial impacto en las cifras de donación y en la opinión pública. Específicamente, por un lado, examinamos las razones que lo respaldan, haciendo especial referencia a aquellas relativas a la utilidad y la justicia. Por otro lado, estudiamos las manifestaciones que se oponen a este modelo teórico fundadas en la vulneración de intereses pre mortem y post mortem, y en el rechazo que generaría en la población. Por último, a modo de conclusión, subrayamos los aspectos que, a nuestro juicio, deberían regularse exhaustivamente si se implantase. En particular, nos referimos al estatuto jurídico del cuerpo humano, al trato al paciente en la última etapa de su vida, a los incentivos para los profesionales sanitarios y al reconocimiento del derecho a la objeción de conciencia.

\section{Palabras clave}

Donación; trasplante; modelo confiscatorio; intereses post mortem; objeción de conciencia; autonomía de la voluntad; calidad de vida; extrapatrimonialidad del cuerpo humano.

\section{Abstract}

This paper analyses the arguments for and against what we have called automatic organ procurement model in relation to the organs of the deceased. For this purpose, this work provides empirical evidence to assess the potential impact of this model on donation rates and on public opinion. Specifically, we examine first the reasons supporting this model, with special reference to utilitarian and justice arguments. On the other hand, we analyse both the approaches based on the violation of pre mortem and post mortem interests opposing this theoretical model and the rejection the model would generate in the population. Finally, we point out the aspects that, in our opinion, should be exhaustively regulated if this model were implemented. In particular, we refer to the legal status of the human body, the treatments for end-of-life patients, the incentives for health professionals and the recognition of the right to conscientious objection

\section{Keywords}

Donation; transplantation; confiscatory model; post mortem interests; conscientious objection; autonomy of will; quality of life; commodification. 


\section{SUMARIO}

I. SITUACIÓN ACTUAL. II. EVALUACIÓN DEL MODELO DE OBTENCIÓN AUTOMÁTICA: 1. Argumentos a favor: 1.1. Razones de utilidad: 1.1.1. Impacto del modelo en las causas de pérdidas de donantes. 1.1.2. Enfermos fallecidos a la espera de un trasplante. 1.2. Razones de justicia. 2. Argumentos en contra: 2.1. La vulneración de la autonomía de la voluntad. Especial referencia a la objeción de conciencia. 2.2. La calidad de los cuidados al final de la vida del potencial donante. 2.3. El rechazo de la población. III. CONCLUSIONES Y REFLEXIONES ACERCA DE UNA PROPUESTA CRÍTICA DE REVISIÓN DE LA NORMATIVA VIGENTE. BIBLIOGRAFIA.

\section{SITUACIÓN ACTUAL}

Prácticamente todos los países del mundo en los que se realizan trasplantes de órganos se rigen por el denominado modelo de donación ${ }^{2}$, que puede adoptar dos formas con múltiples matices: la donación incentivada y la donación sin incentivos (Daar, 1992).

El modelo de donación es el defendido por las principales organizaciones internacionales y por la práctica totalidad de los Estados con base en el principio de extrapatrimonialidad del cuerpo humano (Martin, 2016) y en la idea de que los órganos de cadáver no se pueden obtener en contra de la voluntad (previamente expresada) de la persona fallecida ${ }^{3}$. Sin embargo, tal modelo no consigue abastecer a la totalidad de las personas que necesitan ser trasplantadas, siendo la escasez de órganos un problema creciente con trágicas consecuencias para los enfermos afectados. Ello hace que el modo en el que obtener

2 El término «donación» puede resultar poco adecuado para referirse, entre otras, a la obtención de órganos bajo un sistema de consentimiento presunto (Delgado et al., 2019: 112-118). Para evitar confusiones, queremos aclarar que, en adelante, emplearemos esta expresión («donación») cuando hagamos referencia a los modelos que permiten la obtención de órganos si se cumplen los siguientes dos presupuestos: la ausencia de ánimo de lucro y el respeto a la voluntad del fallecido o de su familia.

3 Los principios de gratuidad y voluntariedad son los que se subrayan en la WHA 63.22 de la OMS de 2010 titulada «Human organ and tissue transplantation» en relación con el modelo de donación. 
órganos para destinarlos a esta terapia constituya uno de los asuntos que más preocupan a las políticas de donación y trasplante, generando un profundo y rico debate bioético. El propósito de este trabajo es explorar las ventajas e inconvenientes de un modelo alternativo al de donación, basado en la obtención automática de órganos de las personas fallecidas, y poder de este modo trazar las líneas básicas de una propuesta de revisión de la normativa vigente (la Ley 30/1979, de 27 de octubre, y el Real Decreto 1723/2012, de 28 de diciembre) a la luz del derecho constitucional a la protección de la salud (art. $43 \mathrm{CE})$.

El Registro Mundial de Trasplantes cifra en 139024 el número total de trasplantes de órganos efectuados en todo el mundo en el año 2018. Este dato contrasta con el número de personas que fallecieron mientras esperaban un trasplante: se estima que ese mismo año fallecieron 3654 enfermos en la Unión Europea. Y las listas de espera de los países que conforman esta organización supranacional albergaban 59845 pacientes a 31 de diciembre de 2018, lo que supone un incremento del $5 \%$ respecto al ańo 2017. Siendo ello así, según la ONT, «apenas se cubren el $10 \%$ de las necesidades de trasplantes en el mundo cifradas por la OMS en más de 1000000 cada año» ${ }^{4}$.

Para hacer frente al insuficiente número de órganos disponibles y ofrecer acceso a esta terapia a un mayor número de enfermos, se han sugerido dos modelos alternativos al modelo de donación: el modelo de mercado y el modelo de obtención automática.

El modelo de mercado ofrece, incluso, un mayor protagonismo al principio de voluntariedad que el modelo de donación. Sus partidarios estiman que los órganos deberían considerarse cosas disponibles y concluyen que todo potencial donante o sus familiares deberían poder hacer con ellos lo que con cualquier otro bien que les pertenezca, sin los límites que se imponen en la actualidad. De este modo, sostienen que se incrementaría la disponibilidad de órganos humanos para trasplantar ${ }^{5}$.

El modelo de mercado podría adoptar dos formas distintas (la de mercado libre y la de mercado regulado) y podría aplicarse para la obtención de órganos tanto de personas vivas como de fallecidos (Shetty, 2009; Matas, 2004; Friedlaender, 2002; Harris y Erin, 2002; Blumstein, 1999). No obstante, la propuesta adquiere mayor aceptación en su versión regulada y cadavérica, al

4 Nota de prensa de la ONT, disponible en: https://bit.ly/34Vgkdm (última consulta: 19 de febrero de 2020).

5 Esta predicción se basa en estudios como el que se realizó para el Journal of Economic Perspectives en el año 2007, en el que se estimó que una compensación de 15000 dólares a cada donante vivo eliminaría la escasez de riñones (Becker y Elías, 2007). 
reducirse, según sus defensores, el riesgo de explotación para el donante y los peligros para su salud. Un mercado regulado de órganos (de vivo) ya se ha implementado en Irán, India y Filipinas (Ghods, 2002; Hippen, 2008; Larijani et al., 2004), con resultados que algunos autores estiman como positivos, al haber reducido e incluso eliminado la lista de espera, y que otros ven con escepticismo por incrementar las desigualdades locales y plantear problemas asociados al turismo de trasplante.

El modelo de obtención automática (a veces referido como «modelo confiscatorio» o "modelo obligatorio») se caracteriza por la ausencia de voluntariedad en la decisión de la extracción post mortem. Su puesta en práctica implica que, una vez fallecida una persona, si alguno de sus órganos fuese útil para realizar un trasplante, se procedería a la extracción de este sin tener en cuenta la voluntad que pudiera haber expresado al respecto ni la de su familia. Si bien el modelo no se ha llevado a la práctica en ningún Estado, su implementación podría llevarse a cabo con indemnización, como sucede con las expropiaciones de terrenos, o sin ella.

El modelo de obtención automática cuenta con el respaldo de algunos autores, sobre todo en relación con la obtención de órganos de fallecidos (Silver, 1989; Fabre, 2006; De Lora, 2012), y genera el rechazo de otros (Rivera, 2001: 69). Este es el motivo por el que un análisis pormenorizado de las promesas y de los retos de esta vía de obtención de órganos resulta tan conveniente.

En este trabajo examinamos con detenimiento cada uno de los argumentos que se manejan a favor y en contra del modelo de obtención automática. Para evitar que los argumentos sobre su posible impacto sean meramente especulativos, nos servimos de extrapolaciones basadas en datos empíricos. Utilizaremos como país de referencia España porque es considerado el país más eficaz en la obtención de órganos trasplantables. Presuponemos que si el modelo de obtención automática fuese el apropiado para dicho Estado, a pesar de ser el país con cifras más altas de donación, entonces ese mismo modelo podría servir, con más razón, para cualquier otro país.

\section{EVALUACIÓN DEL MODELO DE OBTENCIÓN AUTOMÁTICA}

El fundamento teórico del modelo de obtención automática tiene que ver con una política redistributiva de los órganos procedentes de personas fallecidas, según la cual estos serían algo muy similar a los bienes demaniales o de dominio público. Puesto que el ordenamiento jurídico español carece de un pronunciamiento en relación con el estatuto jurídico de las partes del cuerpo 
humano, pueden distinguirse diversas posturas doctrinales: por un lado, se entiende que no son de dominio público porque no pertenecen al Estado. Así pues, o son res nullius o res derelictae o son de propiedad privada (Nicolás, 2006: 339-348). Por otro lado, se sostiene que se trata de bienes privativos, pero que, una vez extraídos del donante, se convierten en bienes de dominio público hasta su trasplante en el receptor (Marina, 2006: 47) .

En este trabajo se asume que los órganos de las personas fallecidas tienen capacidad terapéutica y de salvar vidas, y el Estado podría utilizarlos como recurso en beneficio de la salud pública y el interés general. A continuación, se explica por separado cada uno de los motivos alegados por la doctrina a favor de este modelo y en su contra.

\section{ARGUMENTOS A FAVOR}

Uno de los argumentos más empleados por los defensores del modelo de obtención automática es de corte consecuencialista. En particular, lo que se sostiene es que el coste de su implementación (el posible perjuicio al fallecido y a su familia) es netamente inferior al beneficio que se obtendría en términos de vidas salvadas o mejoradas en su calidad mediante los trasplantes ${ }^{7}$. Esta línea argumental se basa en la afirmación de que la instauración de este sistema llegaría a aumentar el número de órganos disponibles para trasplantar, y evitaría el alto coste de oportunidad asociado al modelo de donación en términos de vidas humanas y de sufrimiento de los pacientes que aguardan en las listas de espera.

6 Fonseca (2010: 190-191), en cambio, no comparte que en el período que transcurre desde que el órgano es extraído al donante hasta que es trasplantado en el receptor este se convierta en un bien de dominio público, ya que, según explica, esta propuesta plantea dos problemas: por un lado, no cumplir el principio de inalienabilidad (que por mandato constitucional debe inspirar el régimen jurídico de los bienes de dominio público), y, por otro lado, no reproducir el juego de los tradicionales instrumentos de la afección y desafección demanial. Por ello, este autor sostiene que más bien parece tratarse de unos bienes vinculados a un determinado destino que se encuentran en una situación de interinidad o dependencia, que desaparecerá una vez determinado el receptor de estos e implantados en dicho sujeto.

7 En el caso del riñón, que es el órgano que más frecuentemente necesita ser reemplazado, el trasplante se considera mejor tratamiento para la insuficiencia renal crónica en cuanto a supervivencia, calidad de vida, complicaciones y relación coste-beneficio que la diálisis (Matesanz y Domínguez-Gil, 2007: 177). 
Un segundo argumento de los partidarios del modelo de obtención automática se basa en la idea de justicia, entendida en términos de reciprocidad: se argumenta que poder recibir un órgano en caso de enfermar y necesitarlo sin a la vez estar dispuesto a ser donante tras la muerte resulta injusto (De Lora y Zúñiga, 2009: 239).

Las razones de utilidad y de justicia son, en definitiva, las que ofrecen el principal sustento del modelo de obtención automática y en las que, secuencialmente, nos detendremos en los siguientes epígrafes.

\subsection{Razones de utilidad}

Entre las razones de utilidad favorables al modelo de obtención automática se ha señalado que, gracias al incremento generado en la oferta de órganos, este modelo contribuiría a eliminar el tráfico de órganos (Moya, 2018: 25-71) y que los sistemas nacionales de salud podrían ahorrarse los costes de las campañas y programas educativos a favor de la donación, así como los derivados de la formación de quienes solicitan los órganos y del mantenimiento de los registros de los donantes (Spital y Erin, 2002; Spital, 2005). Otro argumento consecuencialista empleado ocasionalmente es que ni las familias ni el personal sanitario que realiza la solicitud de órganos necesitarían confrontarse con la decisión, emocionalmente demandante, de proceder o no a la extracción de órganos de una persona recientemente fallecida. Finalmente, contribuiría a la calidad de los órganos extraídos, ya que el proceso de autorización familiar puede prolongarse durante el tiempo, pudiendo amenazar la calidad de los órganos que trasplantar (Spital y Erin, 2002). Sin embargo, el argumento central basado en la utilidad del modelo tiene que ver con su capacidad de salvar la vida de enfermos en lista de espera.

La validez de este argumento depende de que se confirme o refute una hipótesis de carácter empírico: con la implementación del modelo de obtención automática se evitaría la muerte de una parte (o de la totalidad) de los enfermos en lista de espera y se mejoraría la calidad de vida de muchos de los que no están en riesgo vital. Para abordar esta cuestión se precisa conocer, por un lado, el número de órganos que, siendo potencialmente utilizables con objeto de trasplante, no se obtienen actualmente, $y$, por otro, el número de fallecidos a la espera de un trasplante. En los siguientes apartados se realiza la estimación del número de fallecidos actuales en la lista de espera que habrían sobrevivido si se les hubiesen podido trasplantar los órganos no obtenidos por causas que hubiera evitado el modelo de obtención automática. 


\subsubsection{Impacto del modelo en las causas de pérdidas de donantes}

Para averiguar si el modelo de obtención automática tiene la capacidad de incrementar la disponibilidad de órganos con fines terapéuticos, hemos de conocer las causas por las que se pierden potenciales donantes, que en suma, son las siguientes: a) la falta de identificación, b) la falta de mantenimiento de los tratamientos o de las medidas de preservación, c) el rechazo judicial, d) la falta de entrevista familiar, e) el rechazo de la familia a la donación, y f) la ausencia de receptor adecuado.

8 En primer lugar, se pueden perder órganos cuando un potencial donante no es identificado como tal por el equipo de coordinación de trasplantes. Ello sucede, por ejemplo, cuando se detecta erróneamente alguna contraindicación a la donación o cuando se identifica una evolución a muerte cerebral por error y se descarta la donación en asistolia. Al respecto, la ONT ha detectado 10 pérdidas en 2018 por problemas organizativos que podrían encuadrarse en esta primera causa. Véase, en este sentido, el Programa de Garantía de Calidad en el Proceso de Donación de la ONT.

En segundo lugar, la falta de mantenimiento de los tratamientos o de las medidas de preservación del potencial donante también puede ser causa de una pérdida de órganos viables para el trasplante. Las medidas de soporte vital hasta que se produce definitivamente el fallecimiento resultan decisivas para llevar a cabo la donación. Por ende, la no instauración de cuidados intensivos orientados a la donación cuando procede, o la interrupción de estos, será causa de la pérdida de posibilidades de donación. Según el Programa de Garantía de Calidad en el Proceso de Donación de la ONT, hubo 30 pérdidas en 2018 por este motivo.

En tercer lugar, las negativas judiciales, aunque escasas, también suponen pérdidas de órganos. En el año 2018, por ejemplo, hubo 15 negativas judiciales a la obtención de órganos de las 433 consultas judiciales realizadas. Véase, en este sentido, la Memoria sobre actividad de donación y trasplante publicada por la ONT, disponible en: https://bit.ly/3z6Jzb0 (última consulta: 5 de febrero de 2020). Dichas consultas judiciales se realizan en el caso de muertes que sean objeto de investigación judicial. La autorización judicial solo se solicitará si no consta la oposición expresa a la donación por parte del fallecido (art. 5.3 de la Ley 30/1979) y previa diligencia de voluntad de donación para trasplante firmada por un familiar. En este caso no se puede proceder a la extracción de órganos sin la debida autorización judicial por escrito, previo informe del médico forense y siempre que no se obstaculice el resultado de la instrucción de las diligencias penales.

En cuarto lugar, la falta de consulta a la familia también puede configurarse como un factor que derive en una pérdida de órganos viables para trasplante. Esta situación se da en tres circunstancias: cuando el equipo médico no consiga localizar a la familia; cuando, aun siendo localizada aquella, se decide no proceder a realizar la entrevista 
Sin embargo no todas las pérdidas anuales en el potencial de donación son atribuibles al modelo de donación, ni evitables, por lo tanto, con un modelo de obtención automática de órganos. Un sistema que automáticamente obtuviese órganos post mortem para trasplante lograría esquivar solo dos de las cinco causas de pérdidas de órganos viables para trasplante mencionadas (la d y la e).

Mediante la implantación del modelo de obtención automática desaparecería, en definitiva, la necesidad de conocer la voluntad del fallecido, por lo que no podría suponer un obstáculo a la extracción de órganos ni la negativa del fallecido en vida, ni la falta de entrevista familiar, ni la negativa familiar tras la entrevista.

(por ejemplo, si el equipo médico considera que no está preparada para afrontar el proceso de decisión), y cuando se conoce el rechazo del fallecido a la donación por otra vía. En relación con la primera circunstancia, la imposibilidad prolongada de localizar a la familia puede dar lugar a la pérdida de los órganos que están en riesgo de isquemia o a una interrupción de las medidas de preservación de los órganos del donante transcurridas cuatro horas desde el inicio de estas. En el Programa de garantía de calidad de la Organización Nacional de Trasplantes se han contabilizado 5 pérdidas en 2018 por esta causa. Y la tercera circunstancia podría darse, por ejemplo, si se conoce la voluntad del fallecido declarada en vida a través de las voluntades anticipadas registradas. Según datos facilitados por la ONT, de los 2420 casos de donantes en muerte encefálica registrados en 2018, 313 se perdieron por negativa a la donación, habiendo entre ellos 123 negativas del donante en vida.

En quinto lugar, a pesar de lo que establece la legislación española al respecto, que no contempla una exigencia expresa de autorización de la familia para la extracción de los órganos del fallecido (art. 9 RD 1723/2012), puede suceder que la entrevista familiar se lleve a cabo y los órganos no puedan obtenerse debido a su rechazo a la donación. Las negativas familiares a la donación en España en el año 2018, según el Balance de Actividad de la ONT, ascendían al 14,8\%; de 2630 entrevistas realizadas, 391 tuvieron como resultado una negativa a la donación (65 más que en 2017). Véanse, sobre estos datos, la Memoria sobre actividad de donación y trasplante publicada por la ONT y el Newsletter Transplant 2017, del Consejo de Europa, vol. 23, 2018. En este caso pueden producirse dos situaciones: que no se proceda a la instauración de las medidas de mantenimiento del donante o, si aquellas estaban instauradas, que se suspendan.

Por último, en sexto lugar, la ausencia de un receptor adecuado para el órgano también se observa como un obstáculo para un proceso de trasplante exitoso. Según la Memoria de Resultados de la Autoevaluación del año 2018 del Programa de garantía de Calidad del Proceso de Donación de la ONT, ese año, en un total de 44 casos no se inició la extracción de órganos debido a este factor. 
Así pues, el modelo de obtención automática conllevaría la obtención de un mayor número de órganos disponibles para trasplante. Específicamente, en 2018 se hubiesen podido obtener los órganos de los 391 fallecidos cuyos familiares rechazaron la donación y a estos se sumarían los de aquellos fallecidos cuyos familiares no fueron localizados o, habiendo sido contactados, no fueron entrevistados para esta finalidad (cuya cifra se desconoce), así como los de aquellos fallecidos que se opusieron en vida a la donación (124 en 2018).

\subsubsection{Enfermos fallecidos a la espera de un trasplante}

Según el Consejo de Europa, en 2018 fallecieron 10 pacientes europeos al día aguardando un órgano cuando ya estaban incluidos en listas de espera? En España, con una población de 46,4 millones, la Newsletter 2018 del Consejo de Europa, publicado en 2019, cifra en 91 los fallecimientos en lista de espera, de los que 11 fueron de corazón, 25 de pulmón, 51 de hígado, 3 de páncreas y 1 de intestino delgado ${ }^{10}$. Sin embargo, no se hace referencia a la mortalidad estatal en lista de espera de un rińón ${ }^{11}$.

Sobre el dato derivado de la mortalidad a la espera de un riñón solo sabemos que en 2007 el entonces director de la ONT manifestó que «hay más de 5000 pacientes en lista de espera y entre el $8 \%$ y el $10 \%$ de ellos mueren mientras esperan un donante compatible», lo que equivale a una cifra que oscilaría entre los 400 y los 500 fallecidos al año (Pérez, 2008). Y, en la misma dirección, un informe de la Sociedad Española de Nefrología cifró en un $10 \%$ la mortalidad de pacientes en diálisis, pero no especificó qué proporción de estos eran candidatos a trasplante en lista de espera por enfermedad renal crónica avanzada ${ }^{12}$.

$9 \quad$ Nota de prensa de la ONT. Véase la nota n. ${ }^{0} 4$

10 Newsletter Transplant 2018, del Consejo de Europa, vol. 24, 2019.

11 Desconcierta que dicha información no pueda conocerse, teniendo en cuenta que todos los países de nuestro entorno facilitan este dato anualmente (Newsletter Transplant 2018, del Consejo de Europa, vol. 24, 2019). La razón para no facilitar ese dato podría ser que las muertes en lista de rińón no son atribuibles a la ausencia de órgano, ya que existe para todos los enfermos en lista una terapia sustitutiva (la diálisis) y muchas veces la razón por la que muere el paciente es ajena a la enfermedad renal.

12 Véase el Registro de Diálisis y Trasplante renal de la Sociedad Española de Nefrología (Informe 2012-2013). Asimismo, en el Documento de Consenso Nacional de 2012 Donación en Asistolia en España: Situación actual y Recomendaciones, "se objetiva una mortalidad anual en lista del 6 al $8 \%$, lo cual probablemente sea una 
En otros países del entorno, como Francia o Italia, con modelos legislativos y una población análogos, los datos comunicados oficialmente acerca de los fallecidos en lista de espera durante 2018 fueron significativamente superiores a los 91 confirmados por España ${ }^{13}$ : en Francia hubo 676 personas fallecidas (para 65,2 millones de personas) y en Italia, 388 (para 59,3). En ausencia de datos más detallados, solo se puede especular si la diferencia en las cifras de fallecidos se debe solo a la no inclusión de los fallecimientos en lista de espera renal o también a una mayor capacidad del sistema español para abastecer la demanda y disminuir el número de fallecimientos.

Aunque no todos los fallecimientos de enfermos renales en diálisis habrían sido evitados con un trasplante, cabe suponer que el número total de fallecidos en lista de espera en España es superior a 91 ${ }^{14}$. A 31 de diciembre de 2018 había 4804 pacientes en lista de espera. En consecuencia, si, como se estima, la mortalidad anual en lista supera el $8 \%$, los fallecidos en Espańa a la espera de un trasplante habrían sido más de 350 en 2018. Paralelamente, en ese mismo año se contabilizaron en Espańa 391 negativas familiares a la donación $^{15}$, a las que habría que sumar otras pérdidas de órganos aptos para el trasplante derivadas, por ejemplo, de la oposición a la donación por parte del fallecido en vida (124 en 2018). Por lo tanto, si estos órganos se hubiesen podido trasplantar en los receptores más críticos, con todas las cautelas que han de adoptarse en una materia tan sensible como esta, y asumiendo que algunos de estos fallecidos no podrían ser donantes de esos enfermos por contraindicaciones médicas, podrían haberse evitado muchas de esas muertes; quizá la mayoría. Y la misma conclusión alcanzaríamos en relación con otros países en los que el número de rechazos familiares es incluso más elevado que en España. Por ejemplo, en el año 2017 (el último del que tenemos los datos de negativas familiares facilitados por el Newsletter del Consejo de Europa) en Italia hubo 787 rechazos familiares a la donación y 367 fallecidos en lista, por

infraestimación, por cuanto un porcentaje similar de pacientes se excluye cada año de la lista de espera, en muchos casos por un empeoramiento de su situación clínica».

14 Se desconoce tanto en España como en otros países el número de fallecidos previamente excluidos de las listas de espera o de personas candidatas al trasplante que no se incluyen en la lista. En ausencia de estándares internacionales homologados sobre los criterios de inclusión y de exclusión de las listas de espera, resulta imposible ofrecer cifras más exactas sobre el impacto de cualquier política. Las cifras que manejamos son estimativas y, en cualquier caso, conservadoras.

15 Memoria sobre actividad de donación y trasplante publicada por la ONT. Véase la nota n. 8 . 
lo que probablemente se hubiesen podido salvar todas las vidas en riesgo de muerte con la extracción automática de órganos.

En conclusión, una estimación conservadora sugiere que la aplicación de un modelo de obtención automática incrementaría en varios centenares el número de órganos disponibles, pudiendo evitar una cifra nada desdeńable de muertes. En España, esa cifra sería probablemente cercana a la totalidad del número de muertes en lista de espera. Y en otros Estados, como Italia, esa cifra sería incluso superior.

\subsection{Razones de justicia}

El otro argumento principal a favor de un modelo de obtención automática es que tal modelo volvería más justo el sistema actual de distribución de órganos, que permite que ciertas personas puedan ser receptoras de órganos sin que al mismo tiempo estén dispuestas a donar. Los sistemas actuales de donación, basados en el altruismo y la solidaridad, tratan los órganos de cadáver obtenidos con fines de trasplante como un bien público que el Estado distribuye según criterios imparciales como la necesidad o el beneficio esperado. Sin embargo, el empleo de esos criterios acaba beneficiando por igual a quienes cooperan con el sistema (bien sea registrándose como donantes en países con modelo de consentimiento explícito o no oponiéndose a la donación post mortem en los países con modelo de consentimiento presunto) y a quienes no cooperan (oponiéndose a registrar su voluntad de donar o rechazándola explícitamente) pero sí están dispuestos a ser trasplantados en caso de necesitarlo. En un contexto en el que la oferta de órganos se ve ampliamente superada por la demanda, el fenómeno del "polizón» o free rider (personas que se aprovechan de la generosidad de otras personas sin aportar nada a cambio) es entendido por algunos como una injusticia (Zúñiga, 2015: 1334): resulta injusto considerar como potenciales receptores de órganos a quienes se oponen a ser potenciales donantes (De Lora y Zúñiga, 2009: 239; Rivera, 2001: 105-106; Gordillo, 1987: 102).

El modelo de obtención automática no entiende, la justicia en términos de solidaridad, sino de reciprocidad.

A pesar de no haberse llegado a implementar el modelo de obtención automática en ninguna jurisdicción, el principio de reciprocidad no es del todo ajeno a las políticas de trasplante, como las de Israel, Chile o Singapur, que conceden cierta prioridad a los donantes registrados en caso de necesitar un órgano (Zúñiga Fajauri, 2015: 1332-1333). La incorporación del principio de reciprocidad a estas tres legislaciones no ha conseguido, pese a todo, prevenir 
el problema de los free-riders. Esto se debe a que sus normas establecen reglas de priorización, no de exclusión. Por ello, el modelo de obtención automática es para algunos la única manera de erradicar esa injusticia, puesto que ningún beneficiario de los trasplantes podría oponerse a la donación post mortem.

La reciprocidad parece ser uno de los valores que motivan a las personas a donar sangre (Puyol, 2019: 47-48) y órganos (O’Carroll et al., 2019; Ferguson y Lawrence, 2018), lo que podría sugerir que algunas personas sienten la responsabilidad de contribuir a un sistema porque esperan de ese sistema que les ayude en caso de necesitarlo. Sin embargo, no está claro que la reciprocidad sea invocada porque contribuye a un sistema más justo, o simplemente porque contribuye a un sistema más eficaz. Según un estudio llevado a cabo en Canadá, las personas más favorables a la donación valoran efectivamente un modelo basado en la reciprocidad, en el que quienes esperan recibir un órgano deben estar dispuestos a donar. Por el contrario, las personas menos favorables a la donación dudan de que tal sistema pueda promover realmente la donación y cuestionan que contribuya a un sistema más equitativo.

Una objeción a este argumento a favor del modelo de obtención automática es que, como los demás sistemas basados en la reciprocidad, lejos de resolver problemas de justicia, podría incrementarlos, al penalizar a personas que podrían tener razones legítimas para no donar (por ejemplo, personas con discrepancias legítimas sobre el diagnóstico de la muerte o con fuertes creencias post mortem) o a grupos vulnerables de la población cuya motivación para no donar podría estar ligada precisamente a su condición de vulnerabilidad (por ejemplo, grupos de la población con temores fundados a recibir menos del tratamiento debido o a que se anticipe indebidamente el momento de su muerte precisamente por ser considerados como potenciales donantes). Por otro lado, cabría plantearse que la implementación del modelo de obtención automática fuera compatible con un derecho a la objeción de conciencia. A esta posibilidad haremos referencia en las conclusiones.

\section{ARGUMENTOS EN CONTRA}

Las objeciones que pueden plantearse al modelo de obtención automática son también de diversa naturaleza. Sus detractores alegan, en primer lugar, que su aceptación violaría el respeto debido a los intereses post mortem del donante y/o a los deseos de sus seres queridos. En segundo lugar, subrayan que se relajarían las medidas que se deben adoptar para evitar el fallecimiento del potencial donante y para tratarle en la última etapa de su vida con la obligada consideración. Y, en tercer lugar, anticipan que esa política sería rechazada por 
parte de la población, y que generaría desconfianza en el sistema nacional de trasplantes y en el cuerpo médico que lo integra, afectando a otras dimensiones de su práctica.

Seguidamente, se analiza cada uno de estos motivos por separado.

\subsection{La vulneración de la autonomía de la voluntad. Especial referencia a la objeción de conciencia}

Uno de los argumentos más sólidos en contra del modelo de obtención automática es aquel que aduce que resulta inaceptable en cuanto que vulnera la autonomía de la voluntad (Schwark, 2011: 352) o los posthumous interest de las personas afectadas (Glannon, 2003; Hammer y Rivlin, 2003). La consideración de la relevancia de las preferencias previas y los intereses póstumos de los fallecidos puede implicar deberes de dos tipos. Por un lado, un deber directo centrado en el fallecido por la importancia de respetar su autonomía previa y la persona que fue: debemos cumplir sus deseos porque, en el momento de explicitarse, fueron formulados por un ser autónomo. Si negamos esto, no se entiende bien, por ejemplo, por qué respetar las "Instrucciones previas» de un enfermo que ya no recuperará su autonomía, tal y como dispone la Ley 41/2002, de 14 de noviembre. Por otro lado, un deber indirecto, que se relaciona con la esfera del interés público: si no se respetan los deseos de los fallecidos, los vivos sufrirán pensando que nadie cumplirá sus voluntades cuando hayan fallecido. Teniendo presentes estas razones, se acaba concluyendo que existe una obligación moral de respetar los deseos de los difuntos, incluido su interés en no donar sus órganos.

Ahora bien, esta conclusión general, y la creencia en la que se fundamenta (la necesidad de atenerse a los intereses póstumos), merece algunos matices. En primer lugar, como señala Harris (2003), los intereses de los difuntos (critical or persisting interests en su terminología) poseen menos importancia que los de los vivos (experiential interests), incluidos los de las personas enfermas, posibles receptoras de esos órganos. En segundo lugar, este respeto puede conocer límites que no afectan a los de las personas vivas. Estas matizaciones resultan a menudo reflejadas en la normativa vigente en la mayor parte de los ordenamientos jurídicos, que dotan a las autoridades públicas del poder de contravenir los intereses de los difuntos o de sus familiares sobre sus restos mortales si hay razones de interés público que lo avalen. Los dos ejemplos más claros en este sentido son las autopsias tras sospecha de muerte violenta, y la exhumación de cadáveres y posterior autopsia en caso de riesgo epidemiológico. La razón de esta inferencia en el interés de los particulares es fácil de comprender. 
Siguiendo esta lógica, la contravención de los intereses póstumos de los difuntos tiene lugar rutinariamente en nuestras sociedades. Esto no crea una inquietud general ni forja las objeciones que a menudo se presentan en contra de la obtención automática de órganos. Parece que se acepta pacíficamente que, si confluyen ciertas circunstancias, cabe preterir los intereses póstumos de quienes hayan expresado en vida la preferencia de conservar post mortem su integridad corporal. Pero si esto es así en el caso de las autopsias, ¿no debería llegarse a la misma conclusión en lo que atañe a la obtención de órganos? ¿O es que ambos escenarios no son tan similares como pudiera parecer?

La respuesta a esta pregunta ha sido tratada en profundidad por Hershenov y Delaney (2009), quienes identificaron (y posteriormente refutaron) cinco intentos de demostrar por qué una política de autopsias obligatorias es legítima pero la confiscación obligatoria de órganos, no: 1) el contrato social impone al Estado una mayor obligación de proteger a sus ciudadanos frente a los demás que frente a la enfermedad; 2) la prevención de asesinatos constituye una obligación moral superior a la de prevenir muertes causadas por la enfermedad porque matar personas es más grave desde un punto de vista moral que dejar que las personas mueran; 3) las autopsias no confiscan partes del cuerpo, mientras que los trasplantes de órganos sí lo hacen; 4) el hecho de que los ciudadanos sepan que es muy probable que lleguen a confiscar sus órganos generará más ansiedad que la remota posibilidad de una autopsia obligatoria, y 4) una convicción religiosa de que los órganos de uno serán necesarios para resucitar resulta amenazada por el trasplante de órganos, pero no por autopsias que «devuelven» las partes del cuerpo.

Ellos mismos se encargaron de analizar la consistencia de estas objeciones, llegando a unas conclusiones poco alentadoras para quienes las sostienen.

Con respecto a las dos primeras, adujeron en su demérito que es preciso tener en cuenta que las autopsias no solo se realizan en los casos de muertes violentas. Como ya se ha avanzado, las autoridades sanitarias tienen poder suficiente para exhumar los cuerpos de personas sospechosas de haber muerto por una enfermedad infectocontagiosa. En estos casos es obvio que lo que se intenta proteger no es el orden público o la seguridad de no ser asesinado, sino la salud pública. Por consiguiente, las autopsias se realizan pese a la posible oposición de los familiares o de la expresada por el difunto y sin que sea necesario que haya indicios de criminalidad. Así pues, la realización de autopsias en defensa de la salud pública contradice, o por lo menos matiza, las objeciones 1) y 2): ni es cierto que las autopsias se hallen relacionadas con la muerte violenta ni lo es que la necesidad de protegernos frente a la violencia de otros seres humanos sea más acuciante que la de hacer frente a amenazas contra la salud pública.

Con respecto a la tercera, los autores citados argumentan que las autopsias pueden ser más agresivas y causar mayor daño a la integridad corporal que una 
extracción de órganos. Hay, de hecho, ocasiones (como la búsqueda de agentes infecciosos o de según qué venenos) en las que la autopsia puede generar tal deterioro del órgano que este se da por destruido y no se puede devolver al cuerpo. Uno podría pensar que, en todo caso, siempre existirá una diferencia entre un caso y otro, porque en una autopsia solo se deteriora un órgano, mientras que en los casos de obtención de órganos es habitual extraer todos los susceptibles de trasplante, tal y como propone el modelo de obtención automática. Sin embargo, no parece que esta sea una diferencia apreciable para quien se opone a la extracción coercitiva: cualquiera de las dos razones expuestas debe ser aplicable a todos los órganos del cuerpo o no lo será a ninguno, sin opciones intermedias.

En cuarto lugar, Hershenov y Delaney (2009: 378) señalan que la cuestión de la ansiedad conoce muchos matices. Gran parte de la ansiedad que puede generar la posibilidad de que nuestros órganos sean extirpados proviene del desconocimiento de las circunstancias que rodean a la donación. Para empezar, hay quienes creen que ser donante puede incrementar el interés de un facultativo por certificar la muerte para extraer los órganos. Quienes opinan así desconocen, según estos autores, las garantías previstas y la mentalidad del profesional de la medicina, quien, por lo general, antepondrá los intereses de su paciente (el posible donante) a cualquier otro (incluidos los de los posibles receptores). Por consiguiente, es difícil entender la ansiedad que proviene de un temor injustificado: los médicos difícilmente limitarán el esfuerzo terapéutico hacia sus pacientes para beneficio de un tercero con el que no tienen relación alguna.

A esto hay que añadir que, en realidad, lo que debería generarnos ansiedad es la certeza de que, en caso de necesitar un órgano, es posible que no haya ninguno disponible. En tal situación, Hershenov y Delaney concluyen que la imposición de una obligación de donar disminuiría la ansiedad colectiva: aunque pudiésemos sufrir ansiedad pensando que nos extraerán los órganos sin nuestra aquiescencia, este estrés siempre será inferior al que nos suscita un escenario marcado por la falta de órganos con los que satisfacer nuestras necesidades médicas. Esto todavía resulta más plausible si tenemos presente que no es verdad que haya más posibilidades de convertirse en donante de órganos que de sufrir una autopsia ${ }^{16}$.

16 Las cifras más bien dicen lo contrario: por ejemplo, en una sola autonomía española, Murcia, se realizan al año una media de 700 autopsias (véase, al respecto, la Memoria de actividades del Instituto de Medicina Legal y Ciencias Forenses de Murcia, 2018: 69), cifras considerablemente más elevadas que las de donación de órganos, que nunca han superado las 100, según la ONT. Véanse, sobre esto último, los datos históricos de donación de órganos, disponibles en: https://reports.ont.es/datoshistoricos.aspx (última consulta: 27 de febrero de 2020). 
Teniendo, por tanto, todas estas razones presentes, es muy complejo concluir que la autopsia y la obtención automática de órganos posean diferente naturaleza y/o merezcan distinta consideración ética o jurídica. Siendo esto así, la discusión debería centrarse en ponderar si el bien jurídico protegido por un modelo de obtención automática de órganos (la vida o vidas humanas en peligro de muerte) es lo suficientemente relevante como para merecer mayor protección que el bien jurídico protegido por los intereses de los fallecidos (libertad ideológica o de conciencia).

Tal encrucijada fue descrita por Spital y Tailor (2008: 339) en los siguientes términos: "podemos continuar permitiendo a las familias que decidan sobre esto para no exacerbar su situación emocional y para dotarles de la posibilidad de beneficiarse del consentimiento, o podemos sacrificar estos beneficios potenciales en aras de salvar más vidas. Lo que no podemos es tener ambas cosas». En un escenario de este tipo, debemos, a nuestro juicio, tener presente que cuando tratamos con personas en peligro que luchan por vivir, salvar vidas es generalmente la más alta prioridad. $\mathrm{O}$, en palabras de Cronin y Harris (2010: 628), para primar la voluntad del finado, «debemos ser capaces de reconciliarnos con nosotros mismos y dejar claro por qué la libertad de un individuo de controlar su cuerpo después de muerto [...] es más importante que la vida de otra».

Una posible forma de responder al desafío de Cronin y Harris consiste en alejarse de la idea de autonomía o de interés público para apelar a conceptos como la libertad ideológica y de conciencia de las personas (Richards, 2006), un argumento que entronca necesariamente con la quinta objeción a la equiparación entre la autopsia y la obtención automática de órganos identificada por Hershenov y Delaney. La clave en este punto consiste, en general, en subrayar la importancia de esta creencia para algunos grupos religiosos. Se afirma, por ejemplo, que «la tradicional creencia rabínica en la resurrección del cuerpo es, para algunos judíos, la fuente de una importante objeción a la donación de órganos. Ellos creen que el cuerpo debe ser enterrado con todas sus partes, para que estén allí cuando llegue el momento de la resurrección» (Dorff, 1996). Desde otra perspectiva menos ligada a la religión, autores como Glannon (2003) han subrayado que las familias deben poder decidir «cómo encaran el duelo de la muerte de un cónyuge, hermano, hijo o hija» de acuerdo con su visión del mundo, lo que incluye qué sucederá con sus órganos, por ejemplo.

Algunas voces han intentado responder a esta objeción señalando que esto no debería ser un problema porque la mayoría de las religiones no prohíben el uso de órganos cadavéricos, sino que incluso lo fomentan (Schwark, 2011). Sin embargo, este argumento se limita a intentar minimizar 
el efecto de una posible vulneración de intereses fundamentales. Más apropiado resulta aducir que, como todo derecho, la libertad ideológica y de conciencia posee límites, que tienen que ver con el interés o bienestar común, especialmente cuando se trata de la libertad de los difuntos. Por tanto, quien defienda la preponderancia del derecho a la libertad ideológica o de conciencia será quien haya de proporcionar las razones por las que piensa que este debe prevalecer frente a bienes jurídicos como la vida o la salud de terceros (De Lora, 2012).

Con todo, en relación con el modelo de obtención automática, la objeción de conciencia merece una consideración especial. Como se sabe, esta puede ser formulada por un individuo en una situación específica en la que se plantea un conflicto entre un imperativo legal y un imperativo ético (esto es, una convicción, sea religiosa o no). Entonces, si el ordenamiento instaurase el modelo de obtención automática (norma imperativa), cabría plantearse la posibilidad de regular la objeción de conciencia de los profesionales sanitarios. Ahora bien, al tratarse de una norma imperativa de carácter público (la salud pública $)^{17}$, parece incoherente pensar que el legislador fuese a contemplar expresamente la excepción si no la ha previsto en relación con la autopsia.

Además, el problema que presenta este planteamiento es que, en caso de aceptarse, acabaría haciendo del modelo de obtención automática una construcción muy similar a un sistema de consentimiento opt-out, pero eliminando el altruismo que caracteriza a este. La razón es simple. En principio, en un sistema basado en la idea de confiscación, el altruismo no existe: los órganos se donan porque es un deber jurídico. Si aceptamos que es posible incumplir este deber sobre la base de razones ideológicas o religiosas, tenemos dos opciones: o bien obligar al ciudadano a detallar las razones de su objeción, o bien aceptar su negativa sin indagar en su conciencia. Si optamos por lo primero, entraríamos en un juicio de razonabilidad de la conciencia, lo que

Al analizar la jurisprudencia constitucional sobre objeción de conciencia, un sector de la doctrina distingue dos tipos de supuestos: aquellos en los que el conflicto ético se produce con un deber jurídico de carácter privado, en los que sería posible ejercitar la objeción sin necesidad de reconocimiento legislativo previo, por ejemplo, en el marco de una relación contractual, y aquellos otros en los que el conflicto se produce con un deber jurídico de carácter público, en los que para poder ejercitar la objeción de conciencia necesitaríamos el reconocimiento previo de la excepción por parte del legislador: sería el caso de la objeción fiscal, la objeción a participar en mesas electorales o la extinta objeción de conciencia al servicio militar, cuya excepción aparece contemplada en la propia Constitución (Llamazares, 2007: 341-486; Castro, 2016: 441-464). 
parece fuera de lugar. Si, en cambio, optamos por lo segundo, estaríamos permitiendo a cualquiera que lo desease conservar sus órganos quizá solo con cumplir el requisito de inscribirse en una lista de objetores. Pero, de ser así, no parece que el sistema difiera mucho del modelo de donación que rige en la actualidad (sistema opt-out). Luego, debemos concluir que, si estamos dispuestos a reconocer ese derecho a la objeción de conciencia, quizá perdería todo el sentido la misma idea de hablar de un modelo de obtención automática.

En suma, con respecto a la objeción al modelo de obtención automática basada en la idea de intereses póstumos, cabe concluir que la sospecha de la existencia de tales intereses puede justificar el deber prima facie de respetar la memoria y la privacidad de los fallecidos, y la obligación prima facie de honrar el contenido de sus testamentos. Sin embargo, la relevancia moral de los intereses póstumos no es tal que prevalezca sobre las necesidades de los vivos, por lo que solo existirá un deber relativo de respetarlos. Esto es, un deber que solo deberá respetarse estrictamente cuando los intereses póstumos no entren en colisión con intereses que afectan a personas vivas (Banyubala, 2016). Cuando este conflicto surge, esto es, cuando existe un conflicto entre el bienestar de los familiares del fallecido y el de los potenciales receptores de órganos, resulta complicado valorar y comparar el daño que se hace a unos forzándoles a entregar los órganos de sus seres queridos y a otros negándoles una terapia salvadora. Sin embargo, la preferencia de los familiares en contra de la extracción se proyecta (al menos, parcialmente) sobre unos intereses no vitales y, además, ajenos, mientras que la negación del trasplante puede tener unas consecuencias fatales que afectan a los intereses vitales de los receptores, y que experimentan ellos en primera persona, y sus familiares y allegados al verles sufrir o morir. Es cierto que los seres queridos de la persona fallecida pueden sufrir a resultas de la expropiación de los órganos del finado. Es cierto también que habrá personas que sufran pensando que sus intereses póstumos no serán respetados tras su muerte. Pero ese sufrimiento psicológico y moral no parece comparable al daño que sufren tanto el potencial receptor como su familia. Por tanto, la conclusión alcanzada es que ninguna de las razones examinadas en este apartado posee entidad suficiente para justificar una oposición a la obtención automática de órganos.

\subsection{La calidad de los cuidados al final de la vida del potencial donante}

Otra línea de argumentos contrarios al modelo de obtención automática se basa en la eventual vulneración de los intereses de los donantes potenciales 
mientras están vivos. En efecto, aunque el objetivo primario de la obtención de órganos para trasplantes es el de ayudar a vivir a las personas que los necesitan, la consecución de ese objetivo no debe hacerse a costa de los intereses en vida de los donantes potenciales de estos órganos. Por un lado, como cualquier otro paciente, los donantes necesitan protección; es decir, que la posibilidad de que sus órganos pudieran servir para ayudar a otras personas no debería interferir negativamente en la calidad de los cuidados que reciben al final de la vida. En particular, no se debe causar ni precipitar su muerte, ni tampoco causarles ningún tipo de daño o perjuicio que no sufrirían en caso de no ser donantes. Por otro lado, los potenciales donantes necesitan respeto y no deben recibir un trato instrumentalizador por el mero hecho de ser donantes. Concretamente, no se les debe someter a intervenciones fútiles cuyo propósito sea preservar la calidad de los órganos sin contar previamente con su consentimiento o con el de sus allegados. Ahora bien, ciertas medidas necesarias para la consecución del objetivo primario (la maximización del número y calidad de los órganos trasplantables) pueden ser incompatibles con la maximización del objetivo secundario (el respeto y la protección de los donantes). No se trata de un problema específico del modelo de obtención automática, pero puede verse acrecentado por dicho modelo.

La falta de protección de los potenciales donantes de órganos puede darse por varios motivos. En primer lugar, porque los criterios de determinación de la muerte bajo los cuales la extracción de órganos es viable podrían incrementar el número de falsos positivos. En efecto, las dudas que persisten acerca de la validez de los criterios legales actualmente empleados para determinar la muerte, las referidas tanto al criterio neurológico como al criterio cardiorrespiratorio, conducen a algunos autores a la conclusión de que ciertos donantes de órganos podrían estar siendo prematuramente considerados como legalmente muertos (Naïr-Collins, 2015). En segundo lugar, porque los pacientes que son simultáneamente considerados como donantes potenciales pueden ser sometidos a intervenciones fútiles que aceleran o propician su muerte con el único propósito de maximizar la utilidad de un futuro trasplante. Esto sucede, por ejemplo, con pacientes con hemorragia cerebral masiva pero que no cumplen el criterio de muerte encefálica o con pacientes tratados con una craniectomía descompresiva que, una vez que los tratamientos destinados a salvarles la vida han demostrado ser ineficaces, son sometidos a intervenciones poco habituales (como una craneoplastia con vendaje) para que mueran por criterio neurológico, pudiendo de este modo convertirse en donantes multiorgánicos (Rodríguez-Arias et al., 2014). En tercer lugar, no todos los donantes llamados cadavéricos presentan en el momento de la extracción una pérdida total e irreversible de las funciones de 
su cerebro (tales circunstancias no son exigidas en la donación en asistolia), lo que podría implicar, según algunos autores, que esos donantes conserven o puedan recuperar, a resultas de las medidas de preservación, cierta funcionalidad neurológica compatible con la conciencia (Dalle y Bernat, 2018). En estos casos, además de que se les pudiera estar considerando erróneamente como muertos, los pacientes no reciben medidas sedativas en el momento de la extracción de órganos, toda vez que se asume que los cadáveres no pueden sentir (Naïr-Collins, 2017).

En definitiva, posibles faltas de respeto o de protección pueden estar asociadas a las intervenciones pre mortem destinadas a la preservación de la calidad de los órganos. Tales intervenciones no se realizan con un propósito terapéutico ni paliativo, sino que tienen como destinatario último el beneficio de los potenciales receptores de esos órganos. De este modo, la extracción puede implicar un trato instrumentalizador, incluso aunque esa persona no esté siendo dañada. Una falta de respeto ligada a las medidas de preservación podría suceder si tales intervenciones no son consentidas y, sobre todo, si son contrarias a las preferencias de un individuo, a pesar de ser inocuas.

La posibilidad de la obtención de órganos puede generar la percepción, justificada o no, de que los tratamientos debidos a pacientes gravemente enfermos estén siendo comprometidos, restándoles oportunidades de recuperación. Esto podría llevar a la población a desconfiar no solo del sistema de obtención y trasplante de órganos, sino del sistema de salud en general. Algunas personas gravemente enfermas o sus familiares podrían por este motivo negarse a acudir a los hospitales en situaciones críticas, por miedo a que no reciban los tratamientos adecuados o incluso a que se precipite su muerte. Mientras que en un sistema de consentimiento explícito o presunto, estas personas con miedo pueden rechazar ser donantes, no ocurriría así en el marco de un sistema de obtención automática en el que todo el mundo sería donante.

Existen barreras éticas para evitar que los tratamientos a los pacientes gravemente enfermos se vean comprometidos y que las decisiones sobre el final de la vida se vean alteradas por la perspectiva de una donación. Un ejemplo de tal barrera es la exigencia de que en los protocolos de donación en asistolia controlada (tras interrupción del soporte vital) solo se pueda plantear la opción de la extracción una vez que ya se haya decidido que se va a proceder a una limitación del tratamiento de soporte vital.

Por último, cuando los profesionales tienen la múltiple responsabilidad de cuidar a los pacientes ingresados en las unidades de cuidados intensivos, identificar a los donantes potenciales y gestionar las listas de los potenciales receptores de órganos de su hospital, se ven enfrentados a un importante conflicto de 
intereses con consecuencias potencialmente negativas para los pacientes. Los conflictos de interés se caracterizan por un sesgo en el juicio clínico de un profesional, que le puede inducir a subordinar un interés primario (por ejemplo, el beneficio de sus pacientes) por un interés secundario (por ejemplo, el beneficio de los potenciales receptores de sus órganos). La existencia de un conflicto de intereses no garantiza la incurrencia en mala praxis ni la existencia de daños reales a los pacientes. En la actualidad, los daños derivables de los conflictos de intereses están limitados por varias medidas legales y guías de buenas prácticas directamente destinadas a evitarlos, tales como impedir que los equipos de extracción para trasplante participen en el diagnóstico de la muerte encefálica o cardiorrespiratoria y asegurarse de que el proceso de toma de decisiones sobre limitación o retirada del soporte vital sea previo e independiente del proceso de donación. Otra forma menos directa de limitar la posible deriva del conflicto en mala praxis la constituye el requisito de la autorización familiar: si la familia percibe que un equipo médico está condicionando el tratamiento que dan a un ser querido a la perspectiva del beneficio de un trasplante es probable que esta se oponga a la donación. En un modelo de obtención automática no se podría contar con los familiares para que impidieran ciertos abusos resultantes de los conflictos de intereses y de lealtades presentes en los procesos de extracción de órganos para trasplante.

Los obstáculos a los que acabamos de hacer referencia en este epígrafe se han alegado en contra del actual modelo de donación, pero podrían verse incrementados en un modelo de obtención automática, de lo que se concluye que una regulación más estricta sería necesaria para sortearlos.

\subsection{El rechazo de la población}

Lo señalado hasta aquí muestra que existe un debate sobre si la extracción de órganos de las personas declaradas muertas resulta inocua para los intereses de esas personas y sus familiares, pero resulta más difícil argumentar que esos intereses deban sobreponerse a los intereses de las personas vivas potencialmente beneficiarias de sus órganos. Aun si se aceptase esta conclusión favorable a una implantación del modelo de obtención automática, persistiría la objeción de que una política de tal naturaleza podría resultar socialmente disruptiva. ¿Qué impacto social cabe esperar de una política basada en el modelo de obtención automática?

La mayoría de los sistemas actuales de donación incurren en ciertas incongruencias como la de que se siga llamando «donación» a la extracción de órganos que no es explícita sino solo presuntamente consentida o la de que las 
familias tengan la última palabra y puedan desoír las preferencias del fallecido o que en algunos países se pueda retribuir indirectamente a los familiares que autorizan la donación (Rodríguez-Arias et al., 2010). No obstante, los modelos al uso que basan la extracción en las ideas de voluntariedad y altruismo posibilitan que las personas (incluidos los familiares del fallecido) tengan una percepción de control sobre el destino del cuerpo tras la muerte. Ese control se facilita por el hecho de que los potenciales donantes saben que su rechazo será respetado, o porque lo será la oposición de sus familiares, pudiendo estos proteger su interés de no donar llegado el caso. Cabe suponer que para algunas personas tal percepción de control les resulta tranquilizadora, en particular cuando se enfrentan al escenario posible de un fallecimiento próximo, y de forma más general para su relación con el sistema general de salud. Saber que el sistema sanitario no puede apropiarse unilateralmente de los órganos tras la muerte puede contribuir de manera extensiva a la confianza que la ciudadanía tiene con respecto a la medicina y al sistema público de salud. Esta hipótesis parece avalada por el hecho de que una de las principales motivaciones para no donar es la sospecha de que el hecho de ser donante pueda socavar el tratamiento recibido tras haber sido hospitalizado (Shepherd y O'Carrol, 2014; Katsari et al., 2015). Si esto es así, cabe especular que un modelo obligatorio podría no solo infundir temores a las personas que se encuentran próximas a la muerte, sino minar de manera más genérica la percepción social de la relación clínica, y la confianza en el ejercicio de una profesión en la que a los profesionales se les supone y exige una lealtad primaria hacia a sus pacientes.

Apenas existen estudios que permitan saber lo que la población opina acerca del modelo de obtención automática. Una revisión sistemática de la literatura sobre el conocimiento y las actitudes del público hacia modelos de consentimiento para la donación en Europa entre 2008 y 2017 (Molina-Pérez et al., 2019) identificó un único estudio, llevado a cabo en Alemania, que preguntó sobre un sistema de obtención de órganos sin consentimiento (Decker et al., 2008), mostrando que un 38 \% de los encuestados (población general) aceptaría dicho sistema.

Un estudio anterior llevado a cabo en EE. UU. en 2003 (Spital, 2005) preguntó por un sistema de confiscación que incluía la posibilidad de objetar por motivos religiosos: un $31 \%$ de los encuestados se mostraron a favor (de los cuales, un $19 \%$, muy a favor), mientras que un $66 \%$ se mostraron en contra (de los cuales, un $53 \%$, muy en contra).

En Espańa no existen datos publicados acerca de la opinión de la población española al respecto. Por esta razón, hemos llevado a cabo una encuesta en 2018, que enfrentó a 812 personas representativas de la población andaluza a la siguiente afirmación: «A las personas fallecidas se les debería extraer los 
órganos de forma obligatoria, sin tener en cuenta sus preferencias en vida», pidiéndoseles su grado de acuerdo o desacuerdo. El estudio arrojó los resultados siguientes: un 25,6 \% de los encuestados se mostró de acuerdo (de los cuales, un $8,9 \%$, totalmente de acuerdo), mientras que un $68 \%$ se mostró en desacuerdo (de los cuales, un 46,2\%, totalmente en desacuerdo) ${ }^{18}$.

De estos tres estudios podemos sacar la siguiente conclusión: a pesar de que una minoría significativa de la población de Alemania, EE. UU. y España se muestre favorable al modelo de obtención automática, existe un marcado rechazo hacia esa propuesta.

Sin embargo, una encuesta en línea en la que hemos podido participar, llevada a cabo el mismo año 2018 sobre otra muestra de 800 españoles, arroja resultados diferentes (Viciana et al., 2021). En este caso, se planteó el siguiente enunciado:

Considera el tema de los trasplantes de órganos. En España, como en otros países, no hay órganos suficientes para todos los enfermos que los necesitan y que están en lista de espera. Imagina que los médicos estuvieran autorizados a extraer los órganos, para trasplantarlos, de todas las personas fallecidas con órganos viables, sin que a esas personas ni a sus familiares les fuera posible oponerse a la extracción. Ello permitiría salvar vidas de personas que esperan un órgano. En tu opinión, ¿̇debería estar permitido por ley el que, de manera obligatoria, se pudieran extraer los órganos de las personas fallecidas sin su consentimiento o incluso en contra de lo que deseaban y de lo que piden sus familiares?

Los resultados de este estudio indican, por un lado, que un 45,8 \% de los encuestados se muestran a favor y un 54,1\% en contra del planteamiento, y, por otro lado, que a un 51,6 \% de las personas les parece bien y a un 48,3\% les parece mal «la extracción de órganos para trasplantes en las personas fallecidas sin que les sea posible oponerse ni a ellas ni a sus familiares». Además, esta última encuesta permite determinar que aquellos que rechazan el modelo de obtención automática tienen principalmente una justificación deontológica, es decir, basada en el deber moral categórico de respetar la autonomía de las personas, mientras que los que están a favor tienen principalmente una justificación consecuencialista, es decir, apoyada en la idea de que las

18 Encuesta dirigida por D. Rodríguez-Arias y A. Molina Pérez, en colaboración con el IESA-CSIC, que se llevó a cabo en el otoño-invierno de 2018. Resultados todavía no publicados. Comunicación personal. 
consecuencias positivas de este modelo para la sociedad prevalecen sobre otras consideraciones, como el respeto de la autonomía

Ninguna de las encuestas realizadas ofrece detalles acerca de los motivos de rechazo de esa política, por lo que sería oportuno realizar algún tipo de investigación de carácter cualitativo para clarificarlo. A pesar de ello, permiten formular las siguientes hipótesis. En primer lugar, las personas pueden percibir la "confiscación» de órganos como una violación de su autonomía personal, tanto si quieren donar sus órganos como si no. En segundo lugar, muchas personas tienen un sentimiento de propiedad respecto de su cuerpo y del de sus seres queridos, por lo que el modelo de obtención automática podría ser percibido como una expropiación por parte del Estado. En tercer lugar, la donación de órganos es percibida por muchos como un acto de generosidad y altruismo, como un regalo de vida que permite darle algo de sentido a la muerte de un ser querido, mientras que el modelo de obtención automática les privaría de ese sentido. Y, en cuarto lugar, se oponen a la implantación del modelo porque estiman que puede generar un sesgo negativo en el tratamiento de las personas al final de la vida, privándoles, por ejemplo, de determinados esfuerzos clínicos apropiados para mantenerlos con vida.

\section{CONCLUSIONES Y REFLEXIONES ACERCA DE UNA PROPUESTA CRÍTICA DE REVISIÓN DE LA NORMATIVA VIGENTE}

En este trabajo se han examinado los argumentos a favor y en contra del modelo de obtención automática, aportando datos empíricos que permiten valorar su potencial impacto en las cifras de donación y en la opinión del público. De este análisis se desprenden las siguientes conclusiones:

1. El modelo de obtención automática de órganos es una alternativa a los modelos de donación y de mercado, cuya principal característica distintiva es la ausencia de voluntariedad en la decisión de la extracción post mortem.

2. Uno de los principales argumentos a su favor es consecuencialista: incrementaría el número de órganos destinados al trasplante porque se accedería a los que actualmente no se obtienen por rechazo de la familia o del propio donante, y a aquellos, difícilmente cuantificables, por no llevarse a cabo la entrevista familiar para la donación. En España este aumento de la oferta, si atendemos a las estimaciones publicadas por las autoridades espańolas sobre los fallecidos en lista y a los datos facilitados por otros países al respecto, conseguiría evitar, 
en el supuesto menos optimista, la mayoría de muertes de personas a la espera de ser trasplantadas.

3. A ese argumento consecuencialista a favor del modelo de obtención automática se suman otros, también basados en los efectos beneficiosos de su implantación, como el ahorro que supondría prescindir de los programas de promoción de la donación y de la formación de quienes realizan entrevistas familiares, el evitar que algunas familias que preferirían no hacerlo, tengan que decidir sobre el destino de los órganos de su ser querido en un momento tan delicado, y el descenso del tráfico de órganos humanos.

4. El modelo de obtención automática también es defendido por razones de justicia. Al respecto, parece que el modelo más eficaz para evitar el fenómeno del «polizón». El principio de igualdad entendido como reciprocidad sustenta el modelo de obtención automática, pero entra en conflicto con otra comprensión de la justicia, entendida como solidaridad. Hay indicios de que una parte de la sociedad motiva su deseo de donar en la reciprocidad, pero tal motivación no es universalmente compartida ni está claro que se apele a ella por razones de justicia o por razones de utilidad.

5. Entre las objeciones identificadas en contra del modelo de obtención automática destaca la preocupación de que podría atentar contra los intereses póstumos de las personas fallecidas y de sus seres queridos. Sin embargo, parece que, por motivos de interés general y por la relevancia menor de ese tipo de intereses, los intereses póstumos deberían retroceder ante los que verían frustrados los enfermos en lista de espera si no se les trasplantara con esos órganos.

6. Asimismo, existen ciertos riesgos sobre los cuidados al final de la vida del potencial donante que ya se denuncian en el marco del modelo de donación, que se podrían ver incrementados con la implantación del modelo de obtención automática.

7. Por último, los datos más recientes acerca de la opinión de la población sobre los postulados del modelo de obtención automática no ofrecen datos determinantes. Aunque, ciertamente, es aceptado por aproximadamente un $50 \%$ de la población, no puede desconocerse que otro importante sector de la sociedad lo rechaza con rotundidad.

A nuestro juicio los argumentos favorables al modelo de obtención automática parecen ser, en definitiva, más sólidos que los que se oponen a este, si atendemos a las repercusiones del derecho constitucional a la protección de la salud (art. $43 \mathrm{CE}$ ), que debe informar la legislación positiva, la práctica judicial y la actuación de los poderes públicos (art. 53.3 CE). Las principales 
dificultades planteadas radicarían en la puesta en práctica del modelo. En particular, la evidencia sugiere que su implantación podría resultar socialmente disruptiva, al menos para la mitad de la población, y podría generar otro tipo de retos (en particular, por su impacto en las prácticas sobre el final de la vida) y alteraciones en la percepción y la confianza del público en el sistema de salud en general, y en las políticas de donación y trasplante en particular. Por estos motivos sugerimos que esta medida solamente sea explorada mientras continúe el desequilibrio entre la oferta y la demanda de órganos disponibles para trasplantar, considerando las novedades que para el sistema de donación han supuesto la donación en asistolia y la promoción de la donación entre vivos, y tras haber agotado alternativas menos restrictivas de derechos individuales y potencialmente menos onerosas para la estabilidad social, así como para la buena imagen de las políticas de trasplante. Más estudios cualitativos y cuantitativos sobre las implicaciones prácticas y sociales de tal modelo serían también necesarios antes de su implementación. En caso de instaurarse, un pilotaje para reducir su posible impacto social negativo sería altamente aconsejable.

Llegado el caso de que se plantease una reforma legislativa en este sentido, el modelo debería ser regulado, a nuestro juicio, bajo circunstancias estrictas de control y monitoreo, y cumpliendo, además, las siguientes condiciones.

En primer lugar, debería establecerse legalmente el estatuto jurídico del cuerpo humano (de las personas vivas y fallecidas) y de sus partes. Concretamente, resultaría sumamente conveniente establecer, por un lado, si pueden considerarse bienes, y, por otro lado, en caso afirmativo, si serían bienes demaniales, de propiedad privada o si merecerían un tratamiento específico que distinguiese, por ejemplo, los órganos de los vivos de los de los fallecidos, así como los órganos antes de ser extirpados y tras dicho momento.

En segundo lugar, para evitar mala praxis, como, por ejemplo, decisiones prematuras de limitación del esfuerzo terapéutico, la implementación del modelo de obtención automática debería llevarse a cabo sin indemnización para las familias y sin incentivos para los profesionales sanitarios involucrados en la donación vinculados a su rendimiento.

En tercer lugar, el derecho de las personas a elegir las condiciones del final de su vida, dentro del marco regulado para ello, no debería menoscabarse por el cambio al modelo de obtención automática. Así, por ejemplo, debería prevalecer el derecho del paciente a rechazar que le sean prolongados tratamientos fútiles aunque esto pudiera obstaculizar o imposibilitar la finalidad de preservar los órganos y optimizar el trasplante. En este sentido, sería sumamente conveniente potenciar el uso de las instrucciones previas sobre el tratamiento médico en las fases finales de la vida. 
Por último, en cuarto lugar, una adecuada reflexión acerca de la posibilidad de contemplar el reconocimiento del derecho a la objeción de conciencia debería llevar a su rechazo, teniendo en cuenta lo argumentado por el legislador en relación con las autopsias y considerando, además, que su admisión diluiría la razón de ser del modelo de obtención automática frente al modelo de donación que rige en la actualidad.

\section{BIBLIOGRAFÍA}

Banyubala, D. N. (2016). Posthumous Organ Retention and Use in Ghana: Regulating Individual, Familial and Societal Interests. Health Care Analysis, 24 (4), 301-320. Disponible en: https://doi.org/10.1007/s10728-014-0277-4.

Becker Gary, S. y Elías, J. J. (2007). Introducing incentives in the market for live and cadaveric organ donations. Journal of Economic Perspectives, 21 (3), 3-24. Disponible en: https://doi.org/10.1257/jep.21.3.3.

Blumstein, J. (1999). La legalización del pago de órganos de cadáveres para trasplantes. En D. Thomasma y T. Kushner (eds.). De la vida a la muerte. Ciencia y bioética (pp. 136-150). Madrid: Cambridge University Press.

Castro Jover, A. (2016). Libertad de conciencia, objeción de conciencia y derecho a la objeción de conciencia. Quaderni di diritto e politica ecclesiastica, 2, 441-464.

Cronin, A. J. y Harris, J. (2010). Authorisation, altruism and compulsion in the organ donation debate. Journal of Medical Ethics, 36, 627-631. Disponible en: https://doi. org/10.1136/jme.2009.031245.

Daar, A. S. (1992). Rewarded lifting. Transplantation Proceedings, 24, 2201-2211.

Dalle Ave, A. L. y Bernat, J. L. (2018). Inconsistencies between the Criterion and Tests for Brain Death. Journal of Intensive Care Medicine, 1-9. Disponible en: https://doi. org/10.1177/0885066618784268.

Delgado, J., Molina-Pérez, A., Shaw, D. y Rodríguez-Arias D. (2019). The role of the family in deceased organ procurement. Transplantation, 103 (5), 112-118. Disponible en: https://doi.org/10.1097/TP.0000000000002622.

De Lora, P. (2012). El trasplante de órganos y el caso del tranvía: ¿Por qué no confiscamos órganos de cadáver? Jueces para la Democracia, 74, 11-25.

— y Zúñiga, A. (2009). El derecho a la asistencia sanitaria. Un análisis desde las teorías de la justicia distributiva. Madrid: Iustel.

Decker, O., Winter, M., Brähler, E. y Beutel, M. (2008). Between commodification and altruism: gender imbalance and attitudes towards organ donation. A representative survey of the German community. Journal of Gender Studies, 17 (3), 251-255. Disponible en: https://doi.org/10.1080/09589230802204290.

Dorff, E. N. (1996). Choosing Life: Aspects of Judaism Affecting Organ Transplantation. En S. Younger, R. Fox y L. O'Connell (eds.). Organ Transplantation: Meanings and Realities (pp. 168-193). Madison, WI: University of Wisconsin Press. 
Fabre, C. (2006). Whose Body is it Anyway? Justice and the Integrity of the Person. Oxford: Clarendon Press. Disponible en: https://doi.org/10.1093/0199289999.001.0001.

Ferguson, E. y Lawrence, C. (2018). It is only fair: Blood donors are more sensitive to violations of fairness norms than nondonors - converging psychometric and ultimatum game evidence. Vox Sanguinis, 113 (3), 242-250. Disponible en: https://doi.org/10.1111/vox.12636.

Friedlaender, M. (2002). The Right to sell or buy a kidney: are we failing our patients? The Lancet, 359 (9310), 971-973. Disponible en: https://doi.org/10.1016/S01406736(02)08030-3.

Fonseca Ferrandis F. (2010). Trasplantes, calidady administración pública. Granada: Comares. Ghods A. J. (2002). Renal Transplantation in Iran. Nephrology, Dialysis and Transplantation, 17 (2), 222-228. Disponible en: https://doi.org/10.1093/ndt/17.2.222.

Glannon, W. (2003). Do the sick have a right to cadaver organs? Journal of Medical Ethics, 29 (3), 153-156. Disponible en: https://doi.org/10.1136/jme.29.3.153.

Gordillo Cańas, A. (1987). Trasplantes de órganos: 'pietas' familiar y solidaridad humana. Madrid: Civitas.

Hammer, C. L. y Rivlin, M. M. (2003). A Stronger policy for organ retrieval from cadaveric donors: some ethical considerations. Journal of Medical Ethics, 29 (3), 196-200. Disponible en: http://dx.doi.org/10.1136/jme.29.3.196.

Harris, J. (2003). Organ procurement: dead interests, living needs. Journal of Medical Ethics, 29 (3), 130-134. Disponible en: https://doi.org/10.1136/jme.29.3.130.

- y Erin, C. (2002). An ethically defensible market in organ. British Medical Journal, 325, 114-115. Disponible en: https://doi.org/10.1136/bmj.325.7356.114.

Hershenov, D. B. y Delaney, J. J. (2009). Mandatory autopsies and organ conscription. Kennedy Institute of Ethics Journal, 19 (4), 367-391. Disponible en: http://doi. org/10.1353/ken.0.0300.

Hippen, B. E. (2008). Organ sales and moral travails: Lessons from the living kidney vendor program in Iran. Policy Analysis, 614, 1-17.

Katsari, V., Domeyer, P. J., Sarafis, P. y Souliotis, K. (2015). Giving Your Last Gift: A Study of the Knowledge, Attitude and Information of Greek Students Regarding Organ Donation. Annals of Transplantation, 20, 373-380. Disponible en: https://doi. org/10.12659/AOT.894510.

Larijani, B., Zahedi, F. y Taheri, E. (2004). Ethical and legal aspects of organ transplantation in Iran. Transplantation Proceedings, 36 (5), 1241-1244. Disponible en: https:// doi.org/10.1016/j.transproceed.2004.05.065.

Llamazares Fernández, D. (2007). Derecho de la libertad de conciencia. Libertad de conciencia, identidad personal y solidaridad. Navarra: Civitas.

Marina Riopérez, P. (2006). El jurista ante el trasplante de órganos humanos. Granada: Universidad de Granada.

Martin, D. (2016). Trade in kidneys is ethically intolerable. Indian Journal of Medical Ethics, 1 (3), 180-183. Disponible en: https://doi.org/10.20529/IJME.2016.049.

Matas A. J. (2004). The case for living kidney sales: rationale, objections and concerns. American Journal of Transplantation, 4 (12), 2007-2017. Disponible en: https://oi. org/10.1111/j.1600-6143.2004.00664.x. 
Matesanz Acedos, R. y Domínguez-Gil González, B. (2007). Strategies to optimize deceased organ donation. Transplantation Reviews, 21 (4), 177-188. Disponible en: https://doi. org/10.1016/j.trre.2007.07.005.

Molina-Pérez, A., Rodríguez-Arias, D., Delgado-Rodríguez, J., Morgan, M., Frunza, M., Randhawa, G., Reiger-Van de Wijdeven, J., Schiks, E., Wöhlke, S. y Shicktanz, S. (2019). Public knowledge and attitudes towards consent policies for organ donation in Europe. A systematic review. Transplantation Reviews, 33 (1), 1-8. Disponible en: https://doi.org/10.1016/j.trre.2018.09.001.

Moya Guillem, C. (2018). La protección jurídica frente al tráfico de órganos humanos. Especial referencia a la tutela penal en España (art. 156 bis CP). Madrid: Marcial Pons.

Naïr-Collins, M. (2015). Taking science seriously in the debate on death and organ transplantation. Hasting Center Report, 45 (6), 38-48. Disponible en: https://doi. org/10.1002/hast459

- (2017). Can the brain-dead be harmed or wronged?: on the moral status of brain dead and its implications for organ transplantation. Kennedy Institute Ethics Journal, 27 (4), 525-559. Disponible en: https://doi.org/10.1353/ken.2017.0041.

Nicolás Jiménez, P. (2006). La protección jurídica de los datos genéticos de carácter personal. Bilbao-Granada: Comares.

O’Carroll, R., Quigley, J. y Miller, C. B. (2019). The Effect of Reciprocity Priming on Organ Donor Registration Intentions and Behavior. Annals of Behavioral Medicine, 53 (6), 592-595. Disponible en: https://doi.org/10.1093/abm/kay060.

Pérez Oliva, M. (2008). Corazones que laten después de muertos. El País, 7-11-2008. Disponible en: https://bit.ly/3rsGFtP.

Puyol, A. (2019). Ética, solidaridad y donación de sangre. Cuatro perspectivas a debate. Revista de Bioética y Derecho, 45, 47-48. Disponible en: https://doi.org/10.1344/ rbd2019.0.27786.

Richards, A. (2006). Don't Take Your Organs to Heaven... Heaven Knows We Need Them Here: Another Look at the Required Response System. Northern Illinois University Law Review, 26, 365-393.

Rivera López, E. (2001). Ética y trasplante de órganos. México, D.F.: Fondo de Cultura Económica.

Rodríguez-Arias, D., Seoane, J. A., Ramil, C. y Molins, N. (2014). Ethics, new ways of dying and organ donation. Medicina Intensiva, 38 (3), 196-197. Disponible en: https://doi. org/10.1016/j.medin.2013.09.006.

Rodríguez-Arias, D., Wright, L. y Paredes, D. (2010). Success factors and ethical challenges of the Spanish Model of organ donation. The Lancet, 376 (9746), 1109-1112. Disponible en: https://doi.org/10.1016/S0140-6736(10)61342-6.

Schwark, D. (2011). Organ Conscription: How the Dead Can Save the Living. Journal of Law and Health, 24, 323-353.

Shepherd, L. y O'Carroll, R. E. (2014). When do next-of-kin opt-in? Anticipated regret, affective attitudes and donating deceased family member's organs. Journal of Health Psychology, 19 (12), 1508-1517. Disponible en: https://doi. org/10.1177/1359105313493814. 
Shetty, P. (2009). Tax cuts for organs? The Lancet, 374 (9698), 1315-1316. Disponible en: https://doi.org/10.1016/S0140-6736(09)61807-9.

Silver, T. (1989). The case for a Post-Mortem Organ Draft and a proponed Model organ Draft. Boston University Law Review, 68 (4), 681-728.

Spital, A. (2005). Conscription of cadaveric organ for transplantation: a stimulating idea whose time has not yet come. Cambridge Quart Healthcare Ethics, 14 (1), 107-112. Disponible en: https://doi.org/10.1017/S0963180105050127.

- y Erin, C. A. (2002). Conscription of cadaveric organs form transplantation: let's at least talk about it. American Journal of Kidney Diseases, 39 (3), 611-615. Disponible en: https://doi.org/10.1053/ajkd.2002.32164.

- y Taylor, J. S. (2008). In Defense of Routine Recovery of Cadaveric Organs: A Response to Walter Glannon. Cambridge Quarterly of Healthcare Ethics, 17 (3), 337-343. Disponible en: https://doi.org/10.1017/S0963180108080419.

Viciana, H., Hannikainen, I.R. y Rodríguez-Árias, D. (2021). Absolutely right and relatively good: consequentialists see bioethical disagreement in a relativist light. AJOB Empirical Bioethics, 12 (3), 190-205. Disponible en: https://doi.org/10.1080/23294515.2021 .1907476

Zúñiga Fajauri, A. (2015). El consentimiento presunto y la reciprocidad como mecanismos para aumentar la donación. Revista Médica Chile, 143 (10), 1331-1336. Disponible en: https://doi.org/10.4067/S0034-98872015001000012. 